\title{
LA INCIDENCIA DEL CONCEPTO ESTADO DE DERECHO Y ESTADO SOCIAL DE DERECHO EN LA INDEPENDENCIA JUDICIAL
}

\section{Luisa Fernanda García Lozano*}

Fecha de recepción: 14 de Octubre de 2010

Fecha de aceptación: 30 de Noviembre de 2010

Artículo de Reflexión.

\begin{abstract}
Resumen
El siguiente artículo tiene como objetivo realizar un análisis de la incidencia del tipo de Estado en la independencia judicial. En la primera parte, se aborda de forma integral el concepto de Estado de derecho haciendo énfasis en las características históricas y neoinstitucionales que han sido el fundamento de imposición en los últimos años. En la segunda parte, se estudia la trasformación del paradigma jurídico colombiano con su consagración en un Estado Social de Derecho, sus alcances en el campo constitucional $y$, la necesidad de realizar un estudio específico de este tema.
\end{abstract}

\section{Palabras clave}

Estado social de derecho, Estado de derecho, independencia judicial

\section{THE INCIDENCE OF THE CONCEPT OF STATE OF LAW AND SOCIAL STATE OF LAWS IN THE JUDICIAL INDEPENDENCE}

\begin{abstract}
The purpose of this article consists on making an analysis of the incidence of the kind of state on the judicial Independence. Therefor in the first part the concept of state of laws is used with emphasis in the historical and new constitutional characteristics that have been imposed un the last years. The second section studies the transformation of the Colombian law paradigm and its enshrinning in a social state of law, its consequences in the constitutional statement and the necessity to realice a specific study of the subject.
\end{abstract}

\section{Key words}

Social state of law, rule of law, judicial Independence

\footnotetext{
Abogada de la Universidad Santo Tomas, Bogotá; Master in Art of sociology of law Instituto Internacional de sociología Jurídica Oñati, Universidad de País Vasco y Universidad de Milán. Estudiante del doctorado en derecho de la Universidad de Buenos Aires. Ha publicado varios libros en el tema de Sociología Jurídica y Derechos Humanos. Docente - Investigadora. Universidad Santo Tomas, línea de investigación: Derecho y Sociedad, Proyecto de Investigación: El Consejo Superior de la Judicatura: Elementos de Análisis para el estudio de la independencia judicial. luisagarcia@usantotomas.edu.co; elocuencias@yahoo.com
} 


\title{
A INCIDÊNCIA DO CONCEITO ESTADO DE DIREITO E ESTADO SOCIAL DE DIREITO NA INDEPENDÊNCIA JUDICIAL
}

\begin{abstract}
Resumo
Este artigo tem como objetivo fazer uma análise da incidência do tipo de Estado na independência judicial. Na primeira parte, é integralmente abordado o conceito de Estado de direito, dando-se ênfase às características históricas e neo-institucionais que têm sido a base de imposição nos últimos anos. Na segunda parte é estudada a transformação do paradigma jurídico colombiano com sua consagração em Estado Social de Direito, seus alcances no campo constitucional e a necessidade de ser feito um estudo especifico deste tema.
\end{abstract}

Palavras-chave

Estado social de direito, Estado de direito, independência judicial.

\section{INTRODUCCIÓN}

El Estado de Derecho (ED) ha sido por excelencia el tipo de Estado que ha prevalecido en el mundo occidental, de acuerdo a su característica esencial la prevalencia del derecho sobre los aspectos sociales, económicos y políticos, ha sido considerado el máximo protector de los ideales democráticos en la actualidad. Este concepto, con el tiempo, se ha ido transformando y se le ha dado diferentes usos, en la actualidad se considera la base de los valores democráticos y económicos, al igual que una garantía para la defensa de los Derechos Humanos. En el caso Colombiano se encuentra una disyuntiva paradigmática dado que la constitución de 1886 consagro el país como Estado de Derecho - ED, pero en razón de la crisis social, política y económica en 1991, el Estado Social de Derecho- ESD, representará la posibilidad de fortalecimiento de los valores democráticos y sociales. No obstante, en la práctica y en el discurso de los actores del campo jurídico, económico y político los postulados del ED siguen siendo predominantes. Este artículo es una reflexión que parte de una investigación que se está realizando en la universidad Santo Tomas en la cual el problema de investigación consiste en establecer si incide la consagración de una Estado Social de Dere- cho en la Independencia Judicial teniendo como objeto de estudio el Consejo Superior de la Judicatura. Así, el presente texto tiene como objetivo analizar los elementos de cada tipo de Estado, realizar una conceptualización local del tema y su práctica a la luz de elementos constitucionales que permitan hacer un tímido análisis de su incidencia en la independencia judicial, partiendo de una investigación de tipo analítico descriptivo bajo la luz del interrogante ¿el C.S.J. a través de la carrera judicial y el curso concurso ha incidido en el fortalecimiento de la independencia judicial en Colombia?.

Usualmente, en Colombia la corriente que se estudia del nacimiento del ED se ubica en la Revolución francesa al ser este uno de los principales antecedentes del paradigma del derecho aplicado en occidente. De esta forma, la democracia busca prevalecer más allá de las formas de monarquía, en el pueblo reside el poder de control social y este tipo de Estado se establece como la protección a los abusos del poder de las clases dominantes o de los lideres de turno a través de las reglas, la ecuación se hace simple: la reglas, el derecho, es creado por el congreso, este congreso representa el pueblo, por tanto, el ejecutivo y el judicial dependen directamente de esas reglas y acatan su voluntad evitando así la concentración del poder. 
Asimismo, el concepto ingles the rule of law complementa dicha definición dado que en la Carta de dicho país, 1215, se establece la garantía del debido proceso legal como principio fundamental constitucional, el cual dispone que "ningún hombre libre será puesto en prisión, desterrado o muerto sino es mediante un juicio legal de sus pares y conforma a la ley de la tierra o lex terrae"; así, se empieza a gestar uno de los fundamentos de los contextos neoliberales de la posmodernidad al establecer un control de la hegemonía del Estado y del derecho sobre los espacios autónomos a través del procedimiento, (TEUBNER. \& BOURDIEU: 2002) dejando un espacio de acción al derecho sustantivo aspecto que posibilitara un trasformación del concepto ED que brinde poder y fuerza a la independencia judicial.

Así, el ED representa un valor normativo en la práctica institucional al "... generar un ambiente de garantías que haga posible el reconocimiento $y$ goce de las libertades individuales en sus dimensiones negativa y positiva de organizar el poder estatal con el fin de hacer posible la libertad, evitando los riesgos generados por el monopolio de la fuerza que modernamente lo ha caracterizado...". (BURGOS: 2009: 102) Empero, esta acepción implica un imperio de la ley, cualquiera su significado; esto deja de lado la posibilidad que las normas jurídicas no sean socialmente aceptadas, no representen valores sociales o puedan ser manipuladas por el gobernante de turno.

Igualmente, este tipo de Estado representa un control de poder, robustece el legislativo, judicial y ejecutivo como poderes públicos que se regulan entre sí a través de la ley, por ende, “(...) ésta debe ser igualitariamente aplicada por las instituciones estatales que correspondan, incluido el poder judicial. "Igualitariamente", significa que la aplicación administrativa o judicial de las reglas es idéntica para casos equivalentes, que realiza sin tener en cuenta la clase, el status o las asimetrías de poder de los participantes (...dentro del Estado...), y que cumple de conformidad con procedimientos preestablecidos y conocidos"; (O’DONNELL: 2002: 311) así se brinda argumentos formales de protección ciudadana resaltando los principios de seguridad jurídica y validez de la norma.

La conceptualización de este tipo de Estado se desarrolló y ha sido concebida desde "el paradigma de la dogmatica jurídica, la cual se crea en propuestas legales abstractas, impersonales y coercitivas, formuladas por el monopolio de poder público centralizado (el Estado), interpretadas y aplicadas por órganos (poder judicial) y por funcionarios estatales (los jueces)"; (WOLKMER: 2006: 74) entrando en contradicción el significado de poder para la rama judicial, al ser ésta un mero aplicador de las normas preestablecidas sujeto al imperio de la ley.

\section{DESARROLLO DEL ESTADO DE DERECHO}

Para entender la historia del ED en Latinoamérica es necesario acudir a las olas reformistas y el trasplante ideológico que ha impulsado el Banco Mundial. En la época posterior a las dictaduras imperaba la fuerte necesidad de Norte América de usar como "conejillo de indias" a la región, implementando trasformaciones que no estaban acorde con la realidad; (CARVAJAL \& GARCIA: 2006: 265-280) la desorganización de los Estados y la necesidad de garantía de préstamos de organismos internacionales para la construcción de Estados democráticos fueron propicios para efectuar cambios de constituciones e implementar los modelos de Estado. Así, socialmente se inician fuertes luchas en contra de los modelos autoritarios y una posibilidad de iniciar cambios en la región por la débil gobernabilidad histórica de la región.

Los procesos de consolidación de la democracia en Latinoamérica marcaron la necesidad de implementar de manera regional la tercera ola reformista, que consistió en la consolidación de la rama judicial y el fortalecimiento de función de la administración de justicia en varios sentidos. Estos programas iniciaron 
gracias a la agencia de cooperación USAID y varias Universidades Norteamericanas, bajo diferentes tendencias de acuerdo al contexto de cada país, pero encaminados a un mismo fin: "(...) reformar las instituciones de la Rama Judicial bajo los parámetros de fortalecimiento de la democracia y prevalencia del Estado de Derecho". (POPKIN: 2004)

Así, en virtud de los postulados y los contextos del momento se van a empezar a relacionar, en un primer momento, dos conceptos, como son: Democracia y ED, al realizarse una "(...) reflexión sobre la democracia con el Estado de derecho juridificado; este último no sólo, claro está, como texto legal sino, sobre todo, como vigencia efectiva..." lo que hace necesario que la institución encargada de materializar la administración de justicia tomara un papel relevante. Desde esa conceptualización, el tema judicial - lugar privilegiado del enforcementadquiere centralidad debido a que es en ese terreno institucional donde, en definitiva, la igualdad —en cuanto sujetolegal o ciudadanodebe ser efectivamente reconocida; la manera $y$ medida en que lo es, o no, constituye un ambiente que produce determinada densidad democrática", (PASARA: 2003: 415), lo cual permite un orden institucional y un control de los poderes públicos.

Países como Argentina, Chile, Guatemala, Perú, entre otros, tras el proceso de dictadura consagraron su país como Estado de Derecho, lo cual permitió que se implementaran las reformas sin mayores contratiempos y que el modelo económico no estuviera en contraposición con el régimen constitucional; el marco de las trasformaciones políticas, sociales y jurídicas se enmarcaron en el siguiente postulado: “... Si un país no tiene estado de derecho, (...), no será capaz de atraer cantidades sustanciales de inversión extranjera y, en consecuencia, no será capaz de financiar el desarrollo". (CAROTHERS: 2003: 6) En consecuencia, entre las principales preocupaciones que se establecieron por los organismos que inspiraban la tercera ola se encuentra la crisis de credibilidad en la regulación, en la política, la corrupción en las instituciones $e$ inestabilidad de los inversionistas, ${ }^{1}$ por tanto, este organismo procedió a dar directrices de tipo formal para la rama, basadas en la seguridad jurídica resguardando dos principios principalmente la propiedad privada y el cumplimiento de los contratos privados, entonces se configuran como base los siguientes pilares: reglas, procesos e instituciones.

"Reglas: Consiste en reglas objetivas que no sólo son conocidas previamente sino que son verdaderamente ejecutadas y están sujetas a modificación o derogación únicamente por prácticas previamente conocidas.

Procesos: consiste en asegurar que las reglas no sean arbitrarias, o que sean adoptadas en respuesta a necesidades genuinas de la población y aplicadas para servir a estas necesidades.

Instituciones: unas instituciones que funcionen bien y que operen de manera trasparente y sean fiscalizables por los ciudadanos. Instituciones que adhieran y apliquen regulaciones sin arbitrariedad o corrupción. Aquí es dónde el poder judicial entra en acción". (BURGOS: 2009)

Así, se hace evidente que la trasformaciones de finales de la década de los ochenta y comienzos de la década de los noventa, se centran en postulados jurídicos - formales con fines económicos y políticas neoliberales; bajo ese contexto, era necesario el fortalecimiento del derecho y la rama judicial dado que se encontraba que uno de los principales problemas que impidieron la protección de estos derechos y la guarda de la sociedad en general, era su inaplicabilidad en las cortes, así como la falta de memoria histórica al momento de producir los fallos, "(...) La estructura

1 Basado en: BURGOS, G. (2009). Estado de derecho y globalización- El papel del Banco Mundial y las reformas institucionales en América Latina. Bogotá, Colombia: Universidad Nacional de Colombia, Instituto Latinoamericano de Servicios Legales AlternativosILSA. En clave de Sur. 
vertical y autoritaria de los poderes judiciales de la región significaba que los pocos jueces que intentaron ejercer su independencia e investigar casos de violaciones de derechos humanos no encontraron respaldo y fueron vueltos al orden rápidamente. En varios países, jueces que intentaron proteger los derechos de los ciudadanos frente al estado autoritario fueron víctimas de amenazas y, en algunos casos, de atentados", (POPKING: 2004: 411) la rama judicial dependía directamente del ejecutivo y el derecho dictaminaba bajo que parámetros se juzgaba por ende se hacía necesario posterior a fortalecer las reglas de juego, establecer la independencia judicial.

Asimismo, "el banco vincula la división y control de poderes al fin central de estabilizar el marco regulatorio y crear condiciones de credibilidad para los inversionistas. En estos términos, el papel otorgado al poder judicial no concierne a la defensa de determinados derechos ante la extralimitación del poder ,sino a la limitación de la capacidad de decisión de las poblaciones y sus instituciones sobre aspectos económicos fundamentales en su existencia y, de esa forma el poder judicial garantizará un marco de aplicación; es decir, se reviste a los países de reglas de juego a través del principio de seguridad jurídica ,lo cual impide incertidumbre, y genera un ambiente benéfico para los mercados. En relación con este aspecto, el Banco determinó que el poder judicial igualmente debe restringir en los procesos de interpretación de la ley para que sean predecibles los fallos y se consideren eficientes, sobra explicar desde la teoría formal del derecho. (BURGOS: 2009)

No obstante, el proceso que impulsó el Banco Mundial y los organismos de cooperación se enmarcaron en una tendencia que poco a poco se fue apoderando de las teorías económicas, sociológicas y políticas, la cual dio un giro a la perspectiva de la trasplantación y trasformación de procesos: el neoinstitucionalismo. Esta corriente consiste en centrar atención en los cambios organizacionales, e implementación y fortalecimiento de las instituciones. (PORTES:
2006: 13 - 52) Sin embargo, el concepto adoptado principalmente por los economistas para entender las instituciones, tiene componentes muy básicos, éstas se conciben como aquellas influencias externas que influencian el comportamiento de los actores sociales. Esta concepción es bastante general, proviene de la sociología básica pero nos brinda los suficientes elementos de análisis para entender el ejercicio de las instituciones y la teoría de trasplantación ${ }^{2}$.

Con respecto a la independencia judicial, se determinó el uso de un modelo neoinstitucionalista la idea era establecer instituciones y modelos de organización que respondieran a los pilares del estado derecho: reglas, procesos e instituciones; entonces a nivel latinoamericano se trata el tema en virtud de las reformas implementadas de forma institucional u objetiva: creación del Consejo Superior de la Judicatura, carrera judicial, capacitación a jueces y magistrados desde el enfoque del Estado de Derecho, entre otros. Revisar la literatura sobre el tema refleja cómo se determina la independencia judicial a partir de la evidencia de los factores institucionales implementados gracias a los cambios de constitución o reformas que vivió Latinoamérica. (POPKIN: 2004. En: BURGOS: 2004)

Igualmente, los profesores Borja Diaz Rivilla y Sebastian Linares Lejarra, (BORJA \& LINARES: 2005: 89) en el artículo que se titula: "Fortalecimiento de la independencia judicial en Centro América: un balance tras veinte años de reformas" exponen una de las tres investigaciones empíricas presentadas hasta al momento del tema, allí los autores ofrecen tres conclusiones: "En primer lugar, en algunos aspectos fundamentales como asegurar la estabilidad de los jueces las elites nacionales o judiciales no han tenido la voluntad de avanzar lo suficiente. En segundo lugar, el estudio pone de manifiesto que la distancia entre lo que dice la norma y

2 Modelo que toma teorías foráneas y las adaptadas con una filosofía diferente a una necesidad particular, según el contexto geográfico, social, político... 
lo que sucede en la realidad es en muchos casos grande. Por último, el estudio muestra que la construcción del estado de derecho requiere alianzas amplias a favor del cambio y un constante escrutinio de la sociedad civil y de la comunidad donante". Estas conclusiones, evidencian cómo el tema de independencia ha estado ligado a análisis de medición objetivos y que en la práctica es largo el camino que se debe recorrer para que sean eficaces los instrumentos de la independencia en la realidad.

El neoinstitucionalismo ha presentado falencias que no permite tener variables claras para determinar la independencia judicial, más cuando se establecen los avances y las discusiones del tema en dicha perspectiva. Dado que, en palabras de Alejandro Portes:

No hay duda de que 'las instituciones importan', pero están sujetas a lo que Granovetter (1985, 1992) lúcidamente denomina "problema del encaje social": el hechodequelosintercambioshumanos que las instituciones tratan de guiar afectan, a su vez, a estas instituciones. Es por ello que los roles formales y las jerarquías organizativas prescritas llegan a diferir del funcionamiento real de las organizaciones (Dalton 1959, Morrill 1991, Powell 1990). Cuando falta esta separación analítica, así como la comprensión de que las instituciones $y$ organizaciones fluyen de los niveles más profundos de la vida social, todo se convierte en una masa indiferenciada donde el reconocimiento de que 'el con texto importa' produce, a lo sumo, estudios de caso descriptivos y, en el peor de los casos, razonamientos circulares. (PORTES: 2006: 13 - 52)

No obstante, se ha producido otro fenómeno que deviene del Estado de Derecho y ha tenido incidencia en el tema de independencia judicial, "estas son las llamadas fuentes densas que privilegian el aspecto del ED que otorga una mayor capacidad al ciudadano para realizar la vida que desea; en esta perspectiva, se basa de una comprensión expansiva de los derechos civiles, políticos y sociales. La comisión internacional de juristas declaró, "el estado de derecho debería utilizarse no sólo para garantizar y promover los derechos civiles y políticos del individuo en una sociedad libre; sino también para establecer las condiciones sociales, económicas, educativas y su dignidad pueden llegar a hacerse realidad". (RODRÍGUEZ: 2009: 16 - 17) Es decir, ir más allá del modelo neoinstitucional centrando el análisis en la garantía de los derechos, y teniendo como la fuente el respeto de la independencia judicial.

Así, este tipo de ED, tienen su fundamente principalmente en los postulados de Hayek: a) la ley debería ser general, abstracta y prospectiva, de tal modo que el legislador no pueda elegir arbitrariamente a una persona como objeto de su coerción o de su privilegio; b) la ley debe ser conocida y certera, para que los ciudadanos puedan planificar. Para Hayek éste es uno de los factores que más contribuyen a la prosperidad de occidente; c) la ley debe aplicarse a todos los ciudadanos y autoridades oficiales, para que disminuya el incentivo a promulgar leyes injustas; d) debe haber una separación entre los legisladores y quienes tienen el poder de aplicar la ley, los jueces o administradores, de tal modo que las mismas no se creen teniendo en cuenta casos particulares; e) la posibilidad de revisión jurídica sobre las decisiones discrecionales administrativas para corregir eventuales aplicaciones erróneas de la ley; f ) la legislación y la política también deben estar separadas, y la coerción estatal sólo justificada mediante la legislación, para evitar la intimidación de los ciudadanos con propósitos individuales; y g) una Declaración de Derechos no exhaustiva para proteger el ámbito privado. (VILHENA: 2007: 31, 32) Este desarrollo le da una caracterización sustantiva al ED generando parámetros que van más allá de los simples aspectos formales, sin embargo la constante sería una identidad política neoliberalista, se resaltan las características a, c, d y e, dado que estas características empiezan a delinear las 
posibilidades de incidir desde el derecho en aspectos de la vida social más allá de la simple regulación formal.

Esta corriente de ED no centra su fin y desarrollo en la simple regulación, en aspectos formales, como ya se evidenció en los apartes anteriores; la fuente, el fin y el desarrollo del mismo se centra en derechos constitucionales, a este fenómeno se le ha denominado proyecto neoconstitucional global (RODRÍGUEZ: 2009: 18). Su característica principal reposa en un activo control constitucional (COUSO: 2004: 37) y activismo constitucional. (RODRÍGUEZ: 2009: 37)

El control constitucional busca limitar el accionar político y jurídico, por medio de la constitución; de esta forma, se exige que se tenga un fin común en las diferentes ramas del poder, el cual es materializar la constitución. No obstante, al estar presas estas constituciones de contradicciones "el modelo neoliberal versus los derechos fundamentales" se ha explorado la ventaja de los mecanismos de control constitucional al poner límites a las reformas y propuestas netamente neoliberales ,lo cual genera perdida en algunos casos del poder del ejecutivo y el legislativo quienes tienen una representación política.

El activismo constitucional se desprende de la concepción que un $\mathrm{ED}$ debe tener tribunales independientes para mejorar su desarrollo, en este caso en las cortes constitucionales se centra la atención; Por ejemplo, en la creación de ED requiere la existencia de un poder judicial independiente, porque si las normas son razones para las acciones, y el poder judicial es responsable de la aplicación de dichas normas, sería fútil orientar nuestra acción según la ley si los tribunales considerarán otras razones al juzgar. Por el mismo motivo, deben contemplarse los principios del proceso de arreglo, como las audiencias justas o la imparcialidad. El Estado de derecho también requiere que los tribunales tengan el poder para revisar las leyes de otras áreas del gobierno, para garantizar la conformidad con dicho Estado de derecho. Los tribunales deben ser de fácil acceso para no frustrar el Estado de derecho (...)" (VILHENA: 2007: 36). Así, en los países que se implementó el Estado de Derecho Denso, las cortes se apropiaron de la tarea de relegitimar la justicia y el derecho en los diferentes sectores de la sociedad a través de la garantía de los derechos protegidos en la constitución.

Este fenómeno ha sido fuertemente discutido en Latinoamérica, dado que el consenso general concluye que varios de los países de la región han implementado la independencia judicial y su tipo de estado en forma de ED. Para algunos autores, esto ha configurado un uso alternativo del derecho, para otros es sólo un proceso natural de acuerdo a los contextos sociales, políticos y jurídicos que se vivieron en el siglo $\mathrm{XX}$, estos son los casos de Argentina y México en los cuales los tribunales han buscado el fortalecimiento y la protección de los derechos económicos, social y culturales; en estos países, de acuerdo a un estudio realizado por Karina Ansolabehere (ANSOLABEHERE: 2007: 47) se determinó que las cortes constitucionales de ambos países están viviendo un proceso de politización de la justicia. Proceso que bajo el análisis del presente escrito $y$, en virtud que los dos países están consgrados como ED se evidencia trasversalmente el fenómeno de Neoconstitucionalismo en varios niveles, pero que tienen los siguientes lugares comunes: a) la resolución del conflicto relativo a la división de poderes, b) garantía de los derechos civiles, operan condiciones de posibilidad de juego democrático: libertad de asociación, libertad de expresión, libertad de prensa y derecho a la información. Estos países han sido estudiados a profundidad en relación al comportamiento de sus cortes y lo progresista de sus decisiones.

Esta forma de ED se hace relevante en el momento de analizar sus potencialidades y los resultados de las teorías neoinstitucionalistas que se están implementando por parte de las influencias externas de control en los países latinoamericanos. Una de las explicaciones que se encuentran en que los procesos de materialización de cualquiera de los tipos de 
estado confluyen dos aspectos el institucional $y$ el cultural, si bien es cierto en un primer momento sólo quisieron generar marcos formales y prevalencia de la norma en las instituciones, en el proceso de implementación se perdió de vista la fuerte tendencia al respeto a la ley así como a la constitución de los países latinos; por cuanto la tradición jurídica implica un imperio de la ley tanto objetivo como subjetivo, en ese orden de ideas los derechos fundamentales proporcionaron el carácter de garantista y los jueces se adhirieron a éste para dejar de lado la historia de las dictaduras y hacer transito a una democracia.

\section{LA HISTORIA COLOMBIANA: ESTADO SOCIAL DE DERECHO}

A pesar que muchos de los teóricos del tema relacionan han estudiado el caso de Colombia a partir de un EDD y la independencia judicial es considerada un tema exclusivamente del ED, se hace necesario analizar si existen diferencias en el tema de independencia judicial cuando se está consagrado el Estado en Social de Derecho. Es necesario considerar que este análisis se dificulta dado que no existen autores que analicen este tema, relación independencia judicial con el ESD, como anteriormente se hizo referencia el análisis de la independencia judicial se agota en la actualidad en la perspectiva Estado de Derecho Denso. Por lo tanto, la pregunta que convocaría este aparte es ¿̇Existen elementos diferenciales de análisis de la independencia judicial al analizarla con respecto al Estado Social de Derecho?.

En un primer momento, es preciso definir en qué consiste el Estado Social de Derecho ESD-, esté de acuerdo a Heller, se configuró en un principio realizando una limitación a los órdenes económicos liberales para abrir espacio a reivindicaciones sociales en el campo laboral, económico y los órdenes de producción. De esta forma y bajo un proceso de transición se hablaría de un Estado Democrático que hace transito al Estado de Derecho; empero, es necesario reconocer que en el Estado de Derecho bajo el principio social, los derechos serían utilizados integralmente pero se le daría prevalencia a los derechos económicos sociales y culturales. Esta es una concepción clásica de este tipo de Estado, nace después de la segunda guerra mundial y busca contener las políticas salvajes neoliberales para poder volver a recomponer la dignidad humana y los derechos colectivos. Por ende, se estableció una formula en donde el principio fundamental del ED francés, la libertad y la igualdad, principios de la revoluciones sociales se fusionaran brindando mayores garantías. No obstante, estos parámetros son inoperantes en los contextos actuales colombianos sobre todo después de la constitución de 1991, dado que, la historia jurídica Colombiana está compuesta de imposiciones y trasplantes institucionales; esto produjo que dicha Constitución naciera con una fuerte contradicción: Banco Monetario impone políticas neoliberales versus la consagración de Colombia como un ESD.

Igualmente, Carbonell, Orozco y Vázquez refiere tres observaciones para entender este tipo de Estado, las cuales serán analizadas paralelamente con la realidad para determinar de forma más precisa el comportamiento del ESD: "...una, no todo lo que se denomina "imperio de la ley" es necesariamente Estado de Derecho. Esa aseveración puede ejemplificarse con la hipertrofia normativa ("normocracia", diría Heller) de las dictaduras"; sin embargo, Colombia, es un país caracterizado por la superproducción de normas, en algunos casos desconocidas para la comunidad e incluso para los estudiosos del tema, así el exceso ocasiona su ineficacia y en muchos casos se complejizan los asuntos al encontrar múltiples problemas lógicos, parcial- parcial, total, total y parcial total, al momento de su aplicación; "la segunda, que el Estado social de derecho requiere de un "ejecutivo fuerte", capaz de hacer prevalecer el interés reivindicatorio de la sociedad y la aptitud intervencionista del Estado, sobre la vocación complaciente del parlamentarismo", esta característica igualmente en la historia de Colombia ha sido inoperante, dado que 
Colombia se caracteriza por ejecutivos débiles, líderes tradicionales (Weber) que buscan salvaguardar los intereses de las familias que a lo largo del tiempo han mantenido el poder político, social y económico. Así mismo, el último presidente Álvaro Uribe Vélez en sus ocho años de gobierno centró sus políticas en el neoliberalismo y propendió por un discurso de garantías de los derechos políticos desconociendo los económicos, sociales y culturales en perspectiva intervencionista; $y$, la tercera, que existe un evidente parentesco entre el Estado Social de Derecho y el Estado de bienestar. Este último en efecto, suele caracterizarse por la prestación creciente de servicios públicos de interés social como educación, vivienda, abasto, atención médica y asistencia social; un sistema impositivo progresivo; la tutela de los derechos urbano, obrero y agrario, y la redistribución de la riqueza". (CARBONELL, OROZCO \& VÁZQUEZ: 2002: 139) Este aspecto es inaplicable en Colombia, los servicios públicos y de interés social no son garantizados para la población en general, siempre hay un costo, como es el caso de la educación y la atención medica, asimismo, no se han realizado de reformas agrarias, o reformas estructurales que permitan una redistribución de la riqueza, lo cual ha generado y agudizado el conflicto armado y social Colombiano.

Bajo el panorama conceptual anterior expuesto, se hace evidente la necesidad de realizar una reconceptualización del ESD en contextos colombianos, dado que, al analizar los principales elementos de este tipo de Estado fácilmente se pueden contrarrestar con la realidad refutando la eficacia de dichos parámetros. Por ende, para analizar el contexto colombiano, se hace necesario acudir a múltiples fuentes de análisis para poder comprender el concepto de ESD en Colombia y, su aplicación en el tema que convoca como es la independencia judicial.

Colombia para la década de los ochenta, vivía un caos político, jurídico y social; el fortalecimiento del narcotráfico, sus implicaciones con las esferas políticas, el comienzo de la guerra declarada entre las guerrillas y los grupos que después se conocerían como paramilitares, el genocidio de los miembros de la Unión Patriótica, la toma del palacio de justicia por parte del grupo M-19; son sólo algunos de los hechos en el que se sufrió de impunidad, que se evidencio un sistema judicial inoperante, ajeno a la realidad del país, cuyos índices de eficacia y eficiencia eran unos de los más bajos de las últimas décadas.

La crisis y la falta de legitimidad era tal que eran cotidiano encontrar cifras en las cuales "el promedio de negocios por juzgado en Bogotá es de 3.065. En 1983 se acumularon un total de 1.573.300 negocios en la rama penal. En la civil se recubrieron 1.337.000. Un dato más alarmante indica que tramitándose 20.000 procesos por contrabando sólo hay 48 detenidos. Por ende, sistema jurídico más que una garantía de los derechos ciudadanos, más que un mecanismo muy refinado de protección, se ha ido convirtiendo, lenta pero seguramente, en un obstáculo para el desarrollo de nuestras sociedades y en una celestina de los más variados abusos y entuertos y ello por la vía de la impunidad y de la denegación de justicia que se enseñoreó del sistema (...) (CEPEDA: 1985: 65). Cifras como ésta, producían que el derecho estuviera ajeno y la sociedad no legitimara el Estado y, menos la rama judicial.

En un contexto general, las trasformaciones glocales para América Latina incluyendo Colombia iniciaron en esa misma década, como se explicó en el aparte anterior trasformaciones o cambios de la tercera ola reformista, entonces, se fijaron imposiciones de tipo institucional, al igual que económica sin prever las reformas constitucionales en su parte sustantiva; así, el único país que conservaba su carácter democrático para esta época y estaba consagrado a un ED era Colombia, por tal motivo, los movimientos que participaron en la Asamblea Constituyente que tenían como base la trasformación, la lucha contra la desigualdad y la inclusión, propusieron y acataron la idea de dar paso a lo social, al retomar los procesos de lucha que clamaban la reforma, minorías 
étnicas, excluidos, guerrilla, líderes estudiantiles; por tanto, para 1991 se realiza un cambio de Constitución en donde Colombia pasa de ser un Estado de Derecho a un Estado Social de Derecho, se crea un tribunal constitucional y, la posibilidad de acciones públicas asequibles producen cambios en todo el campo jurídico.

Así, el derecho deja de ser concebido como el conjunto de normas instrumentales al servicio en principio del Estado y después de la sociedad, en este sentido, el hecho que en la fórmula del tipo de Estado la sociedad prevalezca sobre el Estado implícito amplia el campo de estudio del significado de lo jurídico, e incluir aspectos políticos y sociales para su materialización.

La definición básica de ESD fue fundada en principios constitucionales como el de la: "dignidad humana, el trabajo, la solidaridad y en la prevalencia del interés general (art1), todo ello complementado con la prevalencia de los derechos inalienables de la persona (arts 5 y 94), la obligación de protección de los débiles, la promoción de igualdad material (art 13), la prevalencia del derecho sustancial sobre los procedimientos (art 228) y la prioridad del gasto publico social (art 350). No obstante, el desarrollo y la interpretación de dichos principios reposarían en principio en entender el derecho como un instrumento y utilizar dichas herramientas desde una apuesta social.

En un primer momento, el desarrollo del concepto de $\mathrm{ESD}$, reposo en las sentencias que emitía la Corte Constitucional, como ya anteriormente se refirió tribunal creado en 1991; la responsabilidad de esta institución era traducir los postulados políticos y jurídicos para brindar un clara directriz de la interpretación y aplicación a futuro de la misma. Comprender la magnitud del significado de este tipo de Estado implica traer a colación tres sentencias que cambiarían la forma de ver el derecho en Colombia. Así, en Sentencia c-449 de 1992, se establece que las instituciones del Estado deben actuar bajo una actitud de servicio hacia la comunidad; bajo esta perspectiva los principios jurídicos en general se enmarcan en esa misma lógica, trasformando la lógica jurídica imperante de la prevalencia del derecho sobre la sociedad M.P Alejandro Martínez Caballero.

Por otro lado, en sentencia T- 406 de 1992, Magistrado ponente Ciro Angarita Varón, explica como la nueva constitución y la consagración a un ESD implicó un cambio en la concepción del derecho, así:[(((..)))] [en] el derecho no sólo hay una transformación cuantitativa debida al aumento de la creación jurídica, sino también un cambio cualitativo, debido al surgimiento de una nueva manera de interpretar el derecho, cuyo concepto clave puede ser resumido de la siguiente manera: "pérdida de la importancia sacramental del texto legal entendido como emanación de la voluntad popular y mayor preocupación por la justicia material y por el logro de soluciones que consulten la especificidad de los hechos". Estas características adquieren una relevancia especial en el campo del derecho constitucional, debido a la generalidad de sus textos y a la consagración que allí se hace de los principios básicos de la organización política. De aquí la enorme importancia que adquiere el juez constitucional en el ESD.

Y por último, en sentencia C- 499 de 1992, magistrado ponente Alejandro Martínez Caballero, cuando realiza un análisis del tema de contratación estatal, ve pertinente hacer reflexiones del tema entorno al ESD, dado que, para dicho magistrado la contratación es un reflejo de la eficacia de las funciones públicas; por lo tanto, en dicha sentencia se determinó: "En un Estado social de derecho las normas le confieren al funcionario un poder reglado en el que se le deja un margen de maniobra o de discrecionalidad al servidor público para que en forma eficaz procure la satisfacción del interés general". Esto, genera una ruptura entre el pensamiento jurídico clásico, dado que los servidores y funcionarios públicos ya no están doblegados a la ley, en sí misma, si no que la base primaria de cualquier actuación del estado estará inmersa en principios sociales. 
En este orden de ideas, las directrices que se constituyeron para este tipo de Estado, tienen como principal fundamento ya no el mantenimiento de la seguridad jurídica, y la legalidad persé; la búsqueda de esta nueva Carta tiene como fin la prevalencia de la justicia social, la legitimidad y la eficacia; características que no poseía Colombia para ese entonces, por ende, en virtud de dichos presupuestos se encuentra como el derecho "deja de ser un límite para el ejercicio del poder y se convierte en un elemento de poder mismo ... al pasar la ley de ser un instrumento de acción... a hacer un instrumento para la acción". (GARCIA, \& UPRIMNY: 2004) En este sentido, los altos tribunales se configuran ya no sólo lo como actores jurídicos, sino políticos incidentes en la realidad nacional al tomar nuevamente fuerza el sistema jurídico en la realidad nacional.

Con miras a lograr los objetivos propuestos por parte de la constituyente y con base en las aspiraciones de la constitución, la eficacia empieza a tener otro significado retomando principios de la sociología jurídica, esta se produce "cuando dispone del consenso activo de sus destinatarios, cuando sea acatado y respetado conscientemente, sin requerir la presión del aparato coercitivo del Estado (derecho legislado) o de la desaprobación por parte de la conciencia común, cuando exista la denominada opinio iuris $\mathrm{u}$ opinio necesitatis, que es la convicción social de la necesidad y la obligatoriedad de la norma", (NOGUERA: 2006: 27) igualmente, se presento el fenómeno por parte de la sociedad civil en exigir un cumplimiento efectivo de la constitución y los mandatos de la Corte produciendo la necesidad de la aplicación real de las normas. Así, gracias a la potestad que brindaron las sentencias referenciadas anteriormente el funcionario, es decir, todo el estado debe trabajar para trasformar la sociedad a una más justa e incluyente.

Asimismo, la legitimidad va a prevalecer sobre la legalidad, es decir, dado que la democracia es uno de los factores que fundamentan este tipo de Estado, la participación ciudadana es conce- bida como uno de los elementos esenciales; sin embargo, el proceso en Colombia funcionó de forma inesperada, la protección a la constitución y su respaldo, legitimidad, se evidenció en virtud de un mecanismo judicial de participación ciudadana, sentencias de constitucionalidad, acción de tutela, acciones populares y de grupo, gracias a estos aspectos se trasfiere algunos trámites y toma de decisiones políticos a los campos judiciales. (UPRIMNY: 2009: 82) Se evidencia entonces, la necesidad de una militancia social para la protección de ese aspecto lo "social" en el Estado, así se legitima su consagración constitucional y genera control social e institucional.

En este sentido y con miras hacer un análisis de la legitimidad en el ESD, se deben analizar el control de constitucionalidad y la acción de tutela como dos herramientas al servicio de la ciudadanía, que han brindado un acceso más próximo a las personas, al igual que un control social acerca de la constitucionalidad de los leyes y decretos en todos los niveles; lo cual, ha generado por parte de la sociedad una protección a estos instrumentos, así como, un reconocimiento de sus funciones.

El control constitucional fue catalogado como uno de los elementos característicos del ESD; sin embargo, en Colombia tiene antecedentes más remotos, éste se consagró en el Acto legislativo numero 3 de 1910, en dicho acto se determinó un control constitucional difuso; pero, sólo hasta 1991 se aplicó este tipo de control. Históricamente se inicia con control concentrado después de 1936 por parte de la Corte Suprema de justicia a las leyes expedidas por el congreso, dado que en la pugna de poderes de esa época el Congreso se volvió sensible frente a las presiones constitucionales y por ende los magistrados fueron tímidos en el ejercicio de dicha función, mientras que los jueces de menor jerarquía preferían la aplicación textual o exegética de las normas. (LÓPEZ: 2006)

El control constitucional representa un límite a los otros poderes públicos y en el contexto colombianolaposibilidad de hacerjusticia social, 
este control se representa básicamente por dos acciones en el caso de la Corte Constitucional: 1. Declaratoria de inconstitucional o inexequibilidad; 2. Inaplicación de la norma por ser inconstitucional en un caso concreto. De esta forma, la judicatura tiene un amplio poder de control, al igual que la potestad de velar por los derechos económicos, sociales y culturales de la población de forma directa; es decir, incidir en los conflictos sociales que se presentan a diario volviendo el derecho un sujeto activo en el campo social. No obstante es necesario que aclarar que no en todo momento las cortes han cumplido a cabalidad con este fin pues para poder adoptar esta postura es necesaria un posición política por parte de los magistrados.

Pese a lo anterior, es necesario advertir como los demás jueces hacen parte del control de constitucionalidad y de la apuesta política, la adopción de la acción de tutela en la Constitución se generó que todos los jueces de cualquier jerarquía iniciaran un acercamiento a la Carta y ejercieran sus funciones de control, así se dio la posibilidad de: "aplicar e interpretar directamente textos constitucionales a casos ordinarios e incluso de inaplicar leyes o normas reglamentarias", (LÓPEZ: 2006: 31) la tutela ha sido uno de los mecanismos por los cuales se ha legitimado directamente el ESD, y la función jurisdiccional del juez al ser los únicos en algunos casos de tener la capacidad de materializar los principios sociales que rigen el país.

La tutela se convirtió en el medio más efectivo para acceder a la administración de justicia, lo que genero el acercamiento de la sociedad, el derecho y sus instituciones. Para 1996 un estudio realizado por el centro de estudios socio- jurídicos de la universidad de los Andes, CIJUS, escrito por Mauricio García Villegas, determinó que las personas que han usado este mecanismo tienen una percepción buena acerca de los usos de esta herramienta, entre los resultados más relevantes dicha investigación, esta que "el $77,6 \%$ de los entrevistados considera que la acción sirve para solucionar la ineficiencia de la justicia”. (GARCIA
VILLEGAS: 1996) Igualmente, en el transcurso de los años la sociedad se ha movilizado para impedir reformas a esta acción, en el 2006 tras la iniciativa de realizar una reforma a la acción de tutela se inicio una polémica sobre el tema, finalmente no se llevo a cabo pero se registró un sondeo de opinión realizado por las páginas web de El Tiempo, Caracol Radio y la Corporación Excelencia en la Justicia, en la cual de 5000 personas el $72 \%$ avaló la tutela como un mecanismo efectivo para la protección de los derechos fundamentales. (EL TIEMPO)

Sí, se analizan los anteriores elementos expuestos para sustentar la transición del régimen colombiano en general, que han permitido la materialización del ESD, estos reposan primeramente en la prevalencia de la constitución y el significado de su contenido, y en un segundo lugar, el aspecto que más interesa para el análisis que se propone, radica en cómo la rama judicial ha sido aquella que ha materializado los derechos sociales, así como el valor de la democracia. Lo que genera el cambio del discurso de la independencia judicial dado que esta característica de la judicatura se convertirá en el elemento que fundamentara el ESD, al volcarse la Rama a la protección de las características antes mencionadas, "la independencia judicial" que era una discusión sesgada en el Estado de Derecho, brindando culto a la ley, toma una connotación relevante al imperar lo social frente a lo jurídico, la única forma de revestir el sistema de seguridad jurídica es a través del aparato judicial.

De esta forma, es necesario evidenciar como la Corte Constitucional siguió la función determinada por el ESD, la caracterización de un juez en este tipo de Estado se caracteriza por "ser creador de principios jurídicos que permitan que el derecho responda a las necesidades sociales" (Sentencia C836 de 2001, Magistrado ponente, Rodrigo Escobar Gil) y, a pesar de la dicotomía que existe entre los derechos fundamentales y la tendencia económica bajo los principios de ESD, tomar decisiones justas e independientes. 
Bajo la anterior lógica de análisis, estudiar las implicaciones del ESD en Colombia pareciese que se agotaran al momento de realizar el análisis económico vs lo social, empero, en varias sentencias la Corte Constitucional agotó el tema y realizo un análisis que determinó el comportamiento del tipo de Estado colombiano de las políticas neoliberales, la sentencia hito Sentencia C-1064 de 2001 M.P.: Manuel Cepeda Espinosa, Jaime Córdoba Triviño, en la cual, se demanda la constitucionalidad de la ley 628 de 2000, art 2 , teniendo como base en sus argumentos donde sostiene que la ley anual de presupuesto para la vigencia fiscal del 1 de enero al 31 de diciembre de 2001, no incluyó las apropiaciones suficientes para aumentar el salario de los empleados públicos en un monto igual o superior a la inflación del año anterior; a pesar de que se declaró exequible el artículo al tener como base el tets de proporcionalidad y la interpretación constitucional para llegar a la igualdad entre los ciudadanos, se establecieron los siguientes criterios para entender el $\mathrm{ESD}$, en este tipo de caso:

El Estado como instrumento de justicia social, basado en una economía social de mercado, con iniciativa privada, pero en la que se ejerce una cierta intervención redistributiva de la riqueza y de los recursos, permite corregir los excesos individuales o colectivistas.

El sistema económico en el Estado social de derecho, con sus características de propiedad privada de los medios de producción, libertad de empresa, iniciativa privada e intervencionismo estatal, está orientado según un contenido humano y por la aspiración de alcanzar los fines esenciales de la organización social. Por ello, el ordenamiento jurídico consagra tanto derechos programáticos, que dependen de las posibilidades presupuestales del país, como derechos prestacionales que dan lugar - cuando se cumplen los requisitos para ello - al ejercicio de un derecho público subjetivo en cabeza del individuo y a cargo del Estado. ...en el Estado Social de Derecho la igualdad material es determinante como principio fundamental que guía las tareas del Estado con el fin de corregir las desigualdades existentes, promover la inclusión y la participación y garantizar a las personas o grupos en situación de desventaja el goce efectivo de sus derechos fundamentales. De esta forma, el Estado Social de Derecho busca realizar la justicia social y la dignidad humana mediante la sujeción de las autoridades públicas a los principios, derechos $y$ deberes sociales de orden constitucional.

\section{A MODO DE CONCLUSIÓN}

Las altas cortes ha sido consideradas independen en la toma de decisiones, sobre todo la Corte Constitucional en sus primeros años y en estos últimos cuatro años la Corte Suprema de Justicia ha adoptado el mismo rol; aunque estas cortes tienen diferencias muy marcadas en sus tendencias ideológicas $y$ en algunas posiciones, es posible evidenciar como algunos casos considerados como hitos constituyen una protección directa a la sociedad, así la independencia judicial ya no es sólo un arreglo de procedimientos que permite una reflexión dentro del mismo derecho para constituirse asimismo, sino por el contrario la parte sustantiva del ESD es el que impera para materializar la independencia. Estas, sentencias han protegido la inclusión de comunidades minoritarias, que han resguardado los intereses generales sobre particulares, que han puesto de presente el carácter social, tribunales que han investigado casos como los de la parapolítica o el fallo contra los crímenes de lesa humanidad, condena de prisión al coronel Plazas por la toma del palacio de justicia ocurrida en 1985; son sólo algunas de las cuales permiten evidenciar cómo a pesar de que en este momento haya un fuerte enfrentamiento entre las políticas del ejecutivo, el legislativo y el judicial , las cortes han propendido por una protección a la consagración del ESD. Por lo tanto, se 
evidencia cómo en relación con las prioridades y el signo político del gobierno se estableció una "Reducción de la capacidad de control del gobierno por parte del poder judicial y ampliación de derechos"4. De Esta forma, se determina cómo la independencia Judicial ha sido un tema fundamental para el análisis de los Estados latinoamericanos; puesto que, en la actualidad, temas, como: la democracia, politización de la justicia, garantía de derechos, son sólo algunos aspectos que trasversalizan las teorías del estado y jurídicas hoy. También, es posible establecer, cómo la transición del tipo de Estado que Colombia vivió ha generado múltiples vacios conceptuales en la aplicación de la Independencia, lo que genera en la literatura actual que el tema sea estudiado solamente a partir del ED impidiendo un desarrollo, profundización y limitando los alcances de la clausula "social" en el tema que nos convoca, siendo este uno de los principales fines del paradigma jurídico posmoderno.

En consecuencia, las reivindicaciones sociales que ha realizado la Rama Judicial en los últimos años se han concentrado en el campo del derecho constitucional que tiene una base política y ampara de forma directa los derechos económicos, sociales y culturales, esto ha generado el análisis desde de la teoría de la politización de la justicia, desconociendo que estas atienden a necesidades sociales e históricas; las cuales, reflejan en las decisiones judiciales un principio de realidad e independencia judicial, consagrado en el preámbulo de la Constitución y son parte del activismo jurídico judicial.

Empero, se hace necesario ampliar el espectro de análisis estudiando: las decisiones de los jueces de

4 Al respecto ver con mayor profundidad el texto: ANSOLABEHERE, K. (2007), Cambios en la política y cambios en la justicia: giro a la izquierda y poder judicial en América Latina. Ciudad de México, México: Fundación Friedrich Ebert (FESMEX). Donde se explica con un análisis comparativo y a través de variables el comportamiento de los tribunales en el periodo de Uribe o Revello - REVELLO, J. (2008, enero - junio). La independencia judicial en tiempos de Uribe. Papel político. Universidad Javeriana, 13, (1), 53 -94 menor jerarquía, el funcionamiento del Consejo Superior de la Judicatura y, los elementos que son indicadores de Independencia a la luz del ESD, y de esta manera determinar cuáles son trasformaciones que se han producido en el estudio y ejecución del derecho bajo las trasformación del tipo de Estado

\section{BIBLIOGRAFÍA}

ANSOLABEHERE, K. (2007), Cambios en la política y cambios en la justicia: giro a la izquierda y poder judicial en América Latina. Ciudad de México, México: Fundación Friedrich Ebert (FESMEX).

BORJA, F. \& LINARES. S. (2005). Fortalecimiento de la independencia judicial en centro América: Un balance tras 20 de años de reformas. (2005). En América Latina Hoy, 39. Salamanca: Universidad de Salamanca.

BURGOS, G. (2009). Estado de derecho y globalización- El papel del Banco Mundial y las reformas institucionales en América Latina. Bogotá, Colombia: Universidad Nacional de Colombia, Instituto Latinoamericano de Servicios Legales Alternativos-ILSA. En clave de Sur.

BURGOS, G. (2009). Justicia bajo presión Constriciones y atentados contra la independencia judicial en Colombia. Bogotá, Colombia: Instituto Latinoamericano de Servicios Legales Alternativos - ILSA.

BURGOS, G. (2004). Independencia judicial en América Latina ¿de quién? ¿Para qué? ¿Cómo?. Bogotá, Colombia: Instituto Latinoamericano de Servicios Legales Alternativos - ILSA. Textos de Aquí y Ahora.

CARBONELL, M., \& OROZCO, W. \& VÁZQUEZ, R. (2002). Estado de derecho, concepto, fundamento y democratización en América latina. Mexico, argentina: Siglo XXI Editores.

CAROTHERS, T. (2003). Promoting The Rule of Law Abroad. The Problem of Knowledge. Washington, EE.UU.: Ruleof Law Series, Carnegie Endowment for International Peace. 
CARVAJAL, J. \& GARCIA, L. (2006) Panorama de las organizaciones de los servicios legales en Colombia. En El otro derecho No 35- El acceso a la justicia, entre el derecho formal y el derecho alternativo. Bogotá, Colombia: Instituto Latinoamericano de Servicios Legales Alternativos-ILSA.

(CARVAJAL \& GARCIA, 2006, pp. 265- 280)

CEPEDA, F. (1985, mayo - junio). Poder judicial y estabilidad democrática. NUEVA SOCIEDAD, Fundación Friedrich Ebert, 77, 65 - 74.

COUSO, J. (2004). Consolidación democrática y poder judicial: los riesgos de la judicialización de la política. En Revista de ciencias políticas, 24, 2. Santiago, Chile: Universidad Católica de Chile.

GARCIA, V. M. \& UPRIMNY, R. (2004). Corte Contitucional y emancipación social en Colombia. En De Sousa Santos B (coord.) Democratizar la democracia. Los caminos de la democracia participativa. Mexico. Fondo de cultura económica.

LÓPEZ, D. (2006). Interpretación constitucional. Bogotá, Consejo Superior de la judicatura, Universidad Nacional. $2^{\mathrm{a}}$ edición.

NOGUERA. A. (2006). Poder y Hegemonía política. El Sistema cruzado de validez y eficacia Derecho-Economía Espiral. Estudios sobre Estado y Sociedad, 37, 11- 48.

O’DONNELL G. (2002). Las poliarquías y la (in)efectividad de la ley en América Latina. En: MENDEZ. J., O' 'DONNELL, G. \& PINHEIRO, P. (comps). La (in)efectividad de la ley y la exclusión en América Latina. Buenos Aires, Barcelona, México: Paidos.

POPKIN M, (2004). Fortalecer la Independencia judicial. En: Pasara, L. (Comp.). En Busca de una justicia distinta: experiencias de reforma en América Latina. Lima, Perú. Consorcio de justicia viva. 409 - 453 .

PORTES, A. (2006). Instituciones y desarrollo una revisión conceptual. Cuadernos de Economía, XXV, (45), 13 - 52.
REVELLO, J. (2008, enero - junio). La independencia judicial en tiempos de Uribe. Papel político. Universidad Javeriana, 13, (1),53 -94.

RODRÍGUEZ, C. (2009). La globalización del estado de derecho. El neoconstitucionalismo, el neoliberalismo y la transformación institucional en América Latina. Bogotá, Colombia: Universidad de los Andes, Centro de estudios CIJUS

TEUBNER, G. (2005). Elementos materiales y reflexivos en el derecho moderno. En La fuerza del derecho. Bogotá, Colombia: Universidad de los andes, Pontificia Universidad Javeriana, instituto pensar, siglo del hombre editores

La judicialización de la política en Colombia: casos, potencialidades y riesgos. En Los jueces: entre el derecho y la política, 81 - 99 .

VILHENA, O. (2007). La desigualdad y la subversión del Estado de derecho. En Sur- revista internacional de derechos humanos. Sao Paulo. Conectas Direitos Humanos (Brasil). El Centro de Derechos Humanos de la Universidad de Pretoria (Sudáfrica) y el Centro de Estudios Legales y Sociales (CELS, Argentina). , 6, 28 - 51.

WOLKMER, A. (2006), Pluralismo Jurídico - Fundamentos de una nueva cultura del derecho. Sevilla, España: Editorial MAD.

\section{Sentencias}

CORTE CONSTITUCIONAL COLOMBIANA. (1992). M.P. CABALLERO, A. Sentencia C-449.

CORTE CONSTITUCIONAL COLOMBIANA. (1992). M.P. CIRO, V. Sentencia T-406.

CORTE CONSTITUCIONAL COLOMBIANA. (1992). M.P. CIRO, V. Sentencia T-499.

CORTE CONSTITUCIONAL COLOMBIANA. (2001). M.P. ESCOBAR, R. Sentencia C-863.

CORTE CONSTITUCIONAL COLOMBIANA. (2001). M.P. CEPEDA, \& CORDOBA, J. Sentencia C-1064. 


\section{Textos en internet}

GARCIA VILLEGAZ. M. (1996). Justicia Constitucional y tutela. Bogotá, Universidad de los Andes, Estudios ocasionales Centro de investigaciones sociojuridicas cijus. [en línea] disponible en: http://cijus.uniandes.edu.co/publicaciones/ publicacionespdf/justiciaconstitucionalyaccion $\% 20$ de\%20tutela.pdf , fecha de consulta, 29 de mayo de 2010.

EL TIEMPO, Caracol Radio y la Corporación Excelencia en la Justicia, la tutela es un mecanismo efectivo para la protección de los derechos fundamentales (revuelo político por la tutela, sábado 22 de abril de 2006, [en línea], disponible en: http:/www.eltiempo.com/ archivo/documento/MAM-1996337"

PASARA, L, (II semestre 2003). Justicia régimen político y sociedad en América Latina. En, Política y gobierno, volumen $\mathrm{X}$, número 2. [En línea], fecha de consulta 13 de mayo de 2010. Disponible en: http://www.politicaygobierno.cide. edu/num_anteriores/Vol_X_N2_2003/Pasara.pdf,

REVUELO POLÍTICO POR LA TUTELA, sábado 22 de abril de 2006, en línea, disponible en: http://www.eltiempo.com/archivo/documento/ MAM-1996337"

\section{Referencias Bibliografícas}

ANSOLABEHERE K. (2007). Cambios en la política y cambios en la justicia: giro a la izquierda y poder judicial en América Latina. Ciudad de México, México: Fundación Friedrich Ebert (FESMEX).

APPENDINI, K. Y NUIJTEN, M. (abril 2002). El papel de las instituciones en contextos locales. Revista de la Cepal. 76, 71- 88.

ARANGO, R. (2003) Corte Contitucional: 10 años, balance y perspectivas. Bogotá, Colombia: Universidad del Rosario.

AYALA, C. (2008). Independencia de la justicia y los procesos constituyentes en la región andina. Estudios Constitucionales, 1, 345 - 348.
BERGALLI, R. (1991, marzo, abril). La quiebra de los mitos, Independencia judicial y selección de los jueces. En Nueva sociedad democracia y política en América - América latina como deuda., 112, 152 - 156.

BERGALLI, R., CORREAS, O. \& FALCÃO, J. ET AL. (1986). Los abogados y la democracia en América Latina, (1ra Ed.). Quito, Ecuador: ILSA - Instituto de Servicios Legales Alternativos.

BURGOS, G. (2007). Independencia judicial: conceptualización y medición. Bogotá, Colombia: Instituto Latinoamericano de Servicios Legales Alternativos - ILSA. Universidad Nacional de Colombia.

BURGOS, G. Justicia Bajo presión Constricciones institucionales y atentados contra la independencia judicial en Colombia 2005 - 2008.

CALDERÓN, F. E. (2003). Análisis normativo, jurisprudencial y fáctico de la corrupción en Colombia 1991-2001. Bogotá, Colombia: Imprenta Nacional.

CARBONELL, M., OROZCO, W. \& VÁZQUEZ, R. (2002). Estado de derecho, concepto, fundamento y democratización en América latina. Mexico, argentina: Siglo XXI editores.

CHINCHILLA, T. (2010). Concepciones sobre el Juez Constitucional en la Reforma de 1910: una cuestión de confianza. En Diálogos de derecho y política, 3. Universidad de Antioquia.

DOMINGO P., SIEDER R. (2001). Rule of law in Latin America: the intenacional promotion of judicial reform. London: Institute of Latin American studies, university of London

DOMINGO, P. Judicialisation of Politics: The Changing Political Role of the Judiciary in Mexico' En SIEDER, R., SCHJOLDEN, L. \& ANGELL, A. (eds). (2005). The Judicialisation of Politics in Latin America. Palgrave.

DROMI, J. (1982, julio). El poder judicial: en la constitución, en la crisis, en la democracia. Tucumán, Argentina: Ediciones UNSTA, Uni- 
versidad del Norte Santo Tomas de Aquino, Católica de Tucumán.

ECHEVERRI, A. Salvamento de Voto En Caso Sittelecom Se Violento La Independencia Judicial.

EVALUACIÓN DE LA GESTIÓN EFICIENCIA Y FINANZAS DE LAS ALTAS CORTES COLOMBIANAS: documento Corte Contitucional.

EVALUACIÓN DE LA GESTIÓN EFICIENCIA Y FINANZAS DE LAS ALTAS CORTES COLOMBIANAS: Corte suprema 2001.

FAJARDO, L., ABONDANO, C. \& GARCÍA, L. (2006), Los invisibles y la lucha por el derecho en Colombia. Una mirada desde las Casas de Justicia, Bogotá, Colombia: Universidad Santo Tomás.

GARCÍA, M. (1993). La eficacia simbólica del derecho: examen de situaciones colombianas. Bogotá, Colombia: Ediciones Uniandes. Universidad de los Andes, facultad de derecho.

GAROT, M. J. (2009), El poder judicial en china cindependiente y eficaz? En: revista para el análisis del derecho, 21 - 20. Barcelona, España: Ministerio de educación, departament d' 'Innovación, universitad empresa.

GLOPPEN, R., GARGARELLA. \& ELIN, S. Ed's. (2004). Democratization and the Judiciary: The Accountability Function of Courts in New Democracies, CASS, F. (2004).

GUARNIERI, C. (2004). Los jueces y la política: poder judicial y democracia. Taurus ediciones, Grupo Santillana.

JUSTICIA Y DESARROLLO DEMOCRÁTICO EN ITALIA Y AMÉRICA LATINA, SEMINARIO DE FORMACIÓN E INFORMACIÓN. (1989, 24 de noviembre, - diciembre)Santo Domingo, República Dominicana.

JUDICIALINDEPENDENCE: The contemporary debate. Shimon shetreet, J. DESCHENES. 1985. Hernández, M. V. (1991). Independencia del juez y desorganización judicial. Madrid, España: Centro de Publicaciones del Ministerio de Justicia, Editorial Civitas.
MARTÍN, J. A. La independencia del poder judicial. Perú.

MOLINA, R. La propuesta de reforma a la elección de magistrados de la Corte Constitucional, en el proyecto gubernamental de reforma a la administración de justicia la imposición de la cooptación.

LÓPEZ, D. (2004). La teoría impura del derecho, Bogotá Colombia: Legis Editores.

LÓPEZ, D. (2006). El derecho de los jueces, (2 ${ }^{\mathrm{a}} \mathrm{Ed}$.). Bogotá, Colombia: Universidad de los Andes, Legis Editores.

OBSERVATORIO COLOMBIANO DE LA ADMINISTRACIÓN DE JUSTICIA. (2005). Independencia en Juego: El caso de la fiscalía nacional de la nación (2001 - 2004). Bogotá, Colombia: Ediciones Antropos.

OFICINA DE DEMOCRACIA Y GOBIERNO (2002), Guidence for promoting judicial independece and imparciality. [Guia para promover la independencia judicial e imparcialidad]. Washington, EE.UU.: Technical Publication Series.

PACHÓN, D. (2008). Ensayos del derecho. Bogotá, Colombia: Universidad INCCA de Colombia. p. $92-110$.

PALACIO, G. Comp. (1989). La irrupción del paraestado. Ensayos sobre la crisis colombiana, Bogotá, Colombia: Fondo Editorial Cerec, Instituto Latinoamericano de Servicios Legales Alternativos.

PÉREZ, C. (2005). Estudios sobre la independencia judicial y el proceso penal. Lima, Perú: Editora jurídica Grijley.

PERUZZOTTI, E. \& SMULOVITZ, C. Enforcing the rule of law: Social Accountability in the New Latin American Democracies. University of Pittsburgh Press.

PORTES, A. (2006). Instituciones y desarrollo una revisión conceptual. Cuadernos de Economía, XXV, (45), p. 13-52.

PRESIDENCIA DE LA REPÚBLICA (1991). Articulo No. 254 - 257 de la Constitución de 
1991, Biblioteca Luis Angel Arango (documento gris)

RAQUEJO, J. L. (1989). Jurisdicción e independencia judicial. Madrid, España: Centro de estudios constitucionales.

SIERRA, D. (2006). El estado de derecho y el imperio del derecho en México durante la transición a la democracia. En Anuario de la facultad de derecho, XXIV, pp. 251-262.

TARAZONA, J. A. (2002). El estado social de derecho y la rama judicial. Bogotá, Colombia: Ediciones Doctrina y Ley.

UPRIMNY, R., \& GARCÍA, M. (2004). Corte Constitucional y emancipación social en Colombia. En Democratizar la democracia: los caminos de la democracia participativa. Fondo de cultura económica. Ciudad de México, México.

UPRIMNY, R. Defensa de la independencia del poder judicial y el principio de la inamovilidad de los jueces- seguridad y estabilidad del poder judicial.

USAID (UNITED STATES AGENCY FOR INTERNATIONAL DEVELOPMENT), OFFICE OF DEMOCRACY AND GOVERNANCE. USAID [AGENCIA DE LOS ESTADOS UNIDOS PARA EL DESARROLLO INTERNACIONAL) OFICINA DE DEMOCRACIA Y GOBIERNO]. (2002), Guidence for promoting judicial independece and imparciality. [Guia para promover la independencia judicial e imparcialidad]. Washington, EE.UU.: Technical Publication Series.

VALDÉS, G. (2003). Independencia judicial y seguridad jurídica Ejes de la convivencia. Bogotá, Colombia: Uno A-Z.

VELÁSQUEZ, C. (2001). Derecho constitucio- nal. Bogotá, Colombia: Universidad Externado de Colombia.

VERDE, R. (2003). El poder judicial en las constituciones de España y Portugal. En Anuario de la facultad de derecho, XXI, pp. 617- 628.

YEPES, H. (1993, septiembre). La Independencia del poder judicial. En Revista Javeriana, 120, (598), p. 247-257.

\section{Textos en internet}

OBSERVATORIO COLOMBIANO DE ADMINISTRACIÓN DE JUSTICIA. (2004). "independencia en entredicho: informe especial sobre la independencia judicial en la fiscalía". www.colectivodeabogados.org/(((..)))/ independencia_judicial.doc

LÓPEZ, D. (2006). Interpretación constitucional. Bogotá, Consejo Superior de la judicatura, Universidad Nacional. (2a Ed.). http://cijus. uniandes.edu.co/publicaciones/publicacionespdf/ justiciaconstitucionalyaccion \%20de\%20tutela. pdf , fecha de consulta, 29 de mayo de 2010

SALAS, L., \& RICO J. Mª La Carrera Judicial en América Latina. [En Línea] Disponible en: www.enj.org. Fecha de consulta 26 de noviembre de 2009

PASARA, L. La cooperación internacional en materia de justicia en el ámbito latinoamericano. [En Línea] Disponible en: http://halshs.archivesouvertes.fr/docs/00/10/41/75/PDF/Pasara.pdf fecha de consulta: 13 de Mayo de 2010

O'DONNELL, G. Accountability horizontal. [En Línea] Disponible en: http://www.insumisos. com/lecturasinsumisas/accountability $\% 20$ democratico.pdf, recuperado: 15 de abril de 2010 